Ferrata Storti Foundation

\title{
A phase 2 study of rituximab, bendamustine, bortezomib and dexamethasone for first-line treatment of older patients with mantle cell lymphoma
}

Haematologica 2019

Volume 104(1):138-146

Parts of this study were presented at the 2014 ASH meeting, the 2017 French Hematology Association meeting and the 2017 International Conference of Malignant Lymphoma.

\section{Correspondence:}

rgressin@chu-grenoble.fr or mary.callanan@chu-dijon.fr

Received: February 23, 2018.

Accepted: August 23, 2018.

Pre-published: August 31, 2018.

doi:10.3324/haematol.2018.191429

Check the online version for the most updated information on this article, online supplements, and information on authorship \& disclosures: www.haematologica.org/content/104/1/138

\section{(C)2019 Ferrata Storti Foundation}

Material published in Haematologica is covered by copyright. All rights are reserved to the Ferrata Storti Foundation. Use of published material is allowed under the following terms and conditions:

https://creativecommons.org/licenses/by-nc/4.0/legalcode. Copies of published material are allowed for personal or internal use. Sharing published material for non-commercial purposes is subject to the following conditions:

https://creativecommons.org/licenses/by-nc/4.0/legalcode, sect. 3. Reproducing and sharing published material for commercial purposes is not allowed without permission in writing from the publisher.

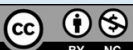

Rémy Gressin ${ }^{1,2}$,Nicolas Daguindau, ${ }^{3}$ Adrian Tempescul, ${ }^{4}$ Anne Moreau, ${ }^{5}$ Sylvain Carras, ${ }^{1}$ Emmanuelle Tchernonog, ${ }^{6}$ Anna Schmitt, ${ }^{7}$ Roch Houot, ${ }^{8}$ Caroline Dartigeas, ${ }^{9}$ Jean Michel Pignon, ${ }^{10}$ Selim Corm, ${ }^{11}$ Anne Banos, ${ }^{12}$ Christiane Mounier, ${ }^{13}$ Jehan Dupuis, ${ }^{14}$ Margaret Macro, ${ }^{15}$ Joel Fleury, ${ }^{16}$ Fabrice Jardin, ${ }^{17}$ Clementine Sarkozy, ${ }^{18}$ Ghandi Damaj, ${ }^{19}$ Pierre Feugier, ${ }^{20}$ Luc Matthieu Fornecker, ${ }^{21}$ Cecile Chabrot, ${ }^{22}$ Veronique Dorvaux, ${ }^{23}$ Krimo Bouadallah, ${ }^{24}$ Sandy Amorin, ${ }^{25}$ Reda Garidi, ${ }^{26}$ Laurent Voillat, ${ }^{27}$ Bertrand Joly, ${ }^{28}$ Philippe Solal Celigny, ${ }^{29}$ Nadine Morineau, ${ }^{30}$ Marie Pierre Moles, ${ }^{31}$ Hacene Zerazhi, ${ }^{32}$ Jean Fontan, ${ }^{33}$ Yazid Arkam, ${ }^{34}$ Magda Alexis, ${ }^{35}$ Vincent Delwail, ${ }^{36}$ Jean Pierre Vilque, ${ }^{37}$ Loic Ysebaert, ${ }^{38}$ Steven Le Gouill,, Mary B. Callanan, ${ }^{2}{ }^{40}$ for the Lymphoma Study Association

${ }^{1}$ Onco-Hematology Department, Grenoble University Hospital; ${ }^{2}$ INSERM 1209, CNRS UMR 5309, Faculté de Médecine, Université Grenoble-Alpes, Institute for Advanced Biosciences, Grenoble; ${ }^{3} \mathrm{Hematology}$ Department, Annecy Hospital; ${ }^{4} \mathrm{Hematology}$ Department, Brest University Hospital; ${ }^{5}$ Pathology Department, Nantes University Hospital; ${ }^{6} \mathrm{Hematology}$ Department, Montpellier University Hospital; ${ }^{7} \mathrm{Hematology}$ Department, Cancer Institute Bergonie Bordeaux; ${ }^{8} \mathrm{Hematology}$ Department, Rennes University Hospital; ${ }^{~} \mathrm{Hematology}$ Department, Tours University Hospital; ${ }^{10} \mathrm{Hematology}$ Department, Dunkerque Hospital; ${ }^{11}$ Hematology Department, Chambery Hospital; ${ }^{12} \mathrm{Hematology}$ Department, Bayonne Hospital; ${ }^{13} \mathrm{Hematology}$ Department, Loire Cancer Institute, Saint Etienne; ${ }^{14}$ Lymphoid Malignancies Unit, Henri Mondor University Hospital, Assistance Publique-Hôpitaux de Paris, Créteil; ${ }^{15}$ IHBN - Hematology Department, Caen University Hospital; ${ }^{16} \mathrm{Hematology} \mathrm{Department,} \mathrm{Clermont-Ferrand}$ Cancer Institute; ${ }^{17} \mathrm{Hematology}$ Department, Rouen University Hospital; ${ }^{18} \mathrm{Hematology}$ Department, Hospices Civils de Lyon, Centre Hospitalier Lyon Sud. INSERM 1052;

${ }^{19} \mathrm{Hematology}$ Department, Amiens University Hospital; ${ }^{20} \mathrm{Hematology}$ Department, Nancy University Hospital; ${ }^{21} \mathrm{Hematology} \mathrm{Department,} \mathrm{University} \mathrm{Hospital} \mathrm{Strasbourg;}$ ${ }^{22} \mathrm{Hematology}$ Department, University Clermont-Ferrand Hospital; ${ }^{23} \mathrm{Hematology}$ Department, Metz University Hospital; ${ }^{24} \mathrm{Hematology}$ Department, Bordeaux University Hospital; ${ }^{25} \mathrm{Hematology}$ Department, University Hospital Paris Saint-Louis; ${ }^{26} \mathrm{Hematology}$ Department, Saint Quentin Hospital; ${ }^{27}$ Hematology Department, Chalon Hospital; ${ }^{28} \mathrm{Hematology}$ Department, Corbeil Hospital; ${ }^{29} \mathrm{Hematology}$ Department, Victor Hugo Clinic, Le Mans; ${ }^{30} \mathrm{Hematology} \mathrm{Department,} \mathrm{Catherine} \mathrm{de} \mathrm{Sienne} \mathrm{Clinic,} \mathrm{Nantes;}$ ${ }^{31} \mathrm{Hematology}$ Department, Angers University Hospital; ${ }^{32} \mathrm{Hematology}$ Department, Avignon Hospital; ${ }^{33}$ Hematology Department, Besançon University Hospital; ${ }^{34} \mathrm{Hematology} \mathrm{Department,} \mathrm{Mulhouse} \mathrm{Hospital;}{ }^{35} \mathrm{Hematology}$ Department, Orleans Hospital; ${ }^{36}$ Onco-Hematology Department, University Hospital Poitiers and INSERM, CIC 1402, Poitiers University; ${ }^{37}$ Hematology Department, Baclesse Caen Cancer Center; ${ }^{38}$ Hematology Department, Toulouse University Hospital; ${ }^{39}$ Hematology Department, Nantes University Hospital and ${ }^{40}$ Unit for Innovation in Genetics and Epigenetics in Oncology, Dijon University Hospital, France

$R G, S L$ and $M B C$ contributed equally to this work.

\section{ABSTRACT}

W e present results of a prospective, multicenter, phase II study evaluating rituximab, bendamustine, bortezomib and dexamethasone as first-line treatment for patients with mantle cell lymphoma aged 65 years or older. A total of 74 patients were enrolled (median age, 73 years). Patients received a maximum of six cycles of treatment at 28-day intervals. The primary objective was to achieve an 18 -month progression-free survival rate of $65 \%$ or higher. Secondary objectives were to evaluate toxicity and the prognostic impact of mantle cell lymphoma prognostic index, Ki67 expression, $\left[{ }^{18} \mathrm{~F}\right]$ fluorodeoxyglucose-positron emission tomography and molecular minimal residual disease, in peripheral blood or bone marrow. With a median follow-up of 
52 months, the 24 -month progression-free survival rate was $70 \%$, hence the primary objective was reached. After six cycles of treatment, $91 \%$ (54/59) of responding patients were analyzed for peripheral blood residual disease and $87 \%$ of these $(47 / 54)$ were negative. Four-year overall survival rates of the patients who did not have or had detectable molecular residual disease in the blood at completion of treatment were $86.6 \%$ and $28.6 \%$, respectively $(P<0.0001)$. Neither the mantle cell lymphoma index, nor fluorodeoxyglucose-positron emission tomography nor Ki67 positivity (cut off of $\geq 30 \%$ ) showed a prognostic impact for survival. Hematologic grade 3-4 toxicities were mainly neutropenia $(51 \%)$, thrombocytopenia (35\%) and lymphopenia (65\%). Grade 3-4 non-hematologic toxicities were mainly fatigue $(18.5 \%)$, neuropathy $(15 \%)$ and infections. In conclusion, the tested treatment regimen is active as frontline therapy in older patients with mantle cell lymphoma, with manageable toxicity. Minimal residual disease status after induction could serve as an early predictor of survival in mantle cell lymphoma. ClinicalTrials.gov: NCT 01457144.

\section{Introduction}

Mantle cell lymphoma (MCL) is a rare subtype of B-cell non-Hodgkin lymphoma characterized by the genetic hallmark $\mathrm{t}(11 ; 14)(\mathrm{q} 13 ; \mathrm{q} 32)$ chromosomal translocation which leads to overexpression of cyclin D1. ${ }^{1}$ The standard-of-care for the treatment of older MCL patients (>65 years), has been eight cycles of R-CHOP (rituximab, cyclophosphamide, doxorubicin, vincristine, and prednisone) given at 21-day intervals (R-CHOP-21), followed by maintenance therapy which has been shown to improve response duration and overall survival (OS) in patients who reach the maintenance phase. ${ }^{2}$ Complete response rates do, however, remain low with R-CHOP $(30-35 \%)$ and the median progression-free survival (PFS) is in the range of 14-18 months. ${ }^{3,4}$ After R-CHOP and maintenance therapy, the 4-year OS rate was $87 \% .^{2}$ Although dose-intensive and high-dose cytarabine-containing regimens, with or without autologous stem cell transplantation consolidation in younger patients, has improved outcomes (the median PFS is now well in excess of 5 years), such approaches are frequently not feasible, given that the median age at diagnosis of MCL is in the mid to late $60 \mathrm{~s} .{ }^{1}$

Bortezomib was the first novel agent to be approved for the treatment of patients with relapsed/refractory MCL. ${ }^{5,6}$ The addition of bortezomib to rituximab-anthracyclinebased regimens has improved the results, compared to those achieved by R-CHOP, for frontline therapy in MCL, leading to a complete response rate of $50 \%$ and a median PFS of 25 months albeit with increased hematologic toxicity. ${ }^{7,8}$ Two phase III trials have shown the superiority of bendamustine-rituximab combination therapy over RCHOP or R-CHOP/R-CVP (rituximab, cyclophosphamide, vincristine, prednisolone) with respect to overall and complete response rates and reduced toxicity.,10 However, superior PFS was observed in only one of the latter phase III studies. ${ }^{9}$ More recently, combining genotoxic agents (such as cytarabine) or targeted agents (such as bortezomib or lenalidomide) with bendamustine and rituximab (BR) has shown efficacy in both first-line and salvage therapy in MCL. ${ }^{1,11-13}$

In anticipation of the above findings, our group initiated a phase II trial to assess the efficacy of a new regimen combining rituximab, bortezomib, bendamustine and dexamethasone (RiBVD) for first-line therapy of older MCL patients. Specifically, for the trial design, associating RiBVD, we took into account the interim results of the $B R$ regimen, for which the reported overall response rates were $90 \%$ in relapsing MCL patients, ${ }^{14,15}$ and the promising results (32\% overall response rate) of bortezomib monotherapy in relapsing MCL patients (PINNACLE study). ${ }^{6}$ Pre-defined secondary objectives of our study included assessment of molecular complete response rates in blood and bone marrow and evaluation of their prognostic impact on survival.

\section{Methods}

\section{Study design and patients}

The RiBVD multicenter phase II trial enrolled newly diagnosed MCL patients $\geq 65$ years or $<65$ years if ineligible or unwilling to undergo autologous stem cell transplantation. The study was conducted in 37 centers of the Lymphoma Study Association (LYSA) (NCT 01457144) and was approved by institutional review boards and ethics committees at all sites, and conducted according to the Declaration of Helsinki. The diagnosis of MCL was established according to the World Health Organization (WHO) 2008 criteria. Ki67 staining and scoring were performed centrally, according to European MCL Network recommendations. ${ }^{16}$ All pathology results were reviewed centrally by the LYSA pathology commission. Eligible patients gave written, informed consent, as per standard guidelines. Inclusion and exclusion criteria are summarized in Online Supplementary Table S1.

The RiBVD regimen consisted of a maximum of six cycles of 28 days each for all enrolled patients, as described in the Online Supplementary Methods and Online Supplementary Table S2.

\section{Response and safety assessments}

The International Working Group (IWG) 1999 and 2007 criteria were used to define responses after four and six cycles, respectively. $\left.{ }^{18} \mathrm{~F}\right]$ fluorodeoxyglucose (FDG)-positron emission tomography (FDG-PET) responses were evaluated in each center with the fivepoint scale, visual method of Deauville. ${ }^{17}$ Hematologic and nonhematologic toxicity was monitored continuously during treatment and at follow-up visits and graded according to the National Cancer Institute criteria (Common Terminology Criteria for Adverse Events, version 3.0) (see the Online Supplementary Methods for details).

\section{Molecular minimal residual disease}

Molecular responses were evaluated centrally by real-time quantitative polymerase chain reaction targeted to patient-specific, IGH V(D)J clono-specific rearrangements, to quantify tumor B cells, according to EURO-MRD guidelines, as previously described. ${ }^{18}$ Minimal residual disease (MRD) analysis was per- 
formed before treatment (baseline), after four courses of treatment (mid-term MRD), and at the end of treatment (after 6 courses of RiBVD) in peripheral blood and bone marrow until progression or relapse, for a maximum follow-up period of 3 years. ${ }^{18,19}$ During the follow-up, MRD was evaluated in the blood at 3 monthly intervals for 1 year and every 6 months thereafter while bone marrow $\mathrm{MRD}$ monitoring was performed at yearly intervals. A description of additional methods, the MRD study cohort, sample source and numbers is given in the Online Supplementary Methods and illustrated in Online Supplementary Figure S1.

\section{Sample size calculation and statistical analysis}

The primary objective of the study was to prolong PFS by 6 months compared to the 18-month median PFS reported for patients treated with R-CHOP-21. ${ }^{3}$ The number of patients to be enrolled was calculated by a one-step Fleming method. In order to define superiority of the RiBVD regimen over R-CHOP, a PFS rate of $65 \%$ or more $(\mathrm{H} 1)$ was required at 18 months. The treatment was to be considered a failure if the PFS rate at 18 months was $\leq 50 \%$. Taking into account alpha and beta risks of $5 \%$ and $20 \%$, respectively, 69 patients needed to be enrolled. Based on a maximum $10 \%$ error in diagnosis, 76 patients had to be enrolled. Additional details are given in the Online Supplementary Methods.

\section{Results}

\section{Patients}

A total of $76 \mathrm{MCL}$ patients were enrolled between November 2011 and December 2012 (Figure 1). All patients were monitored for 3 years after their last cycle of

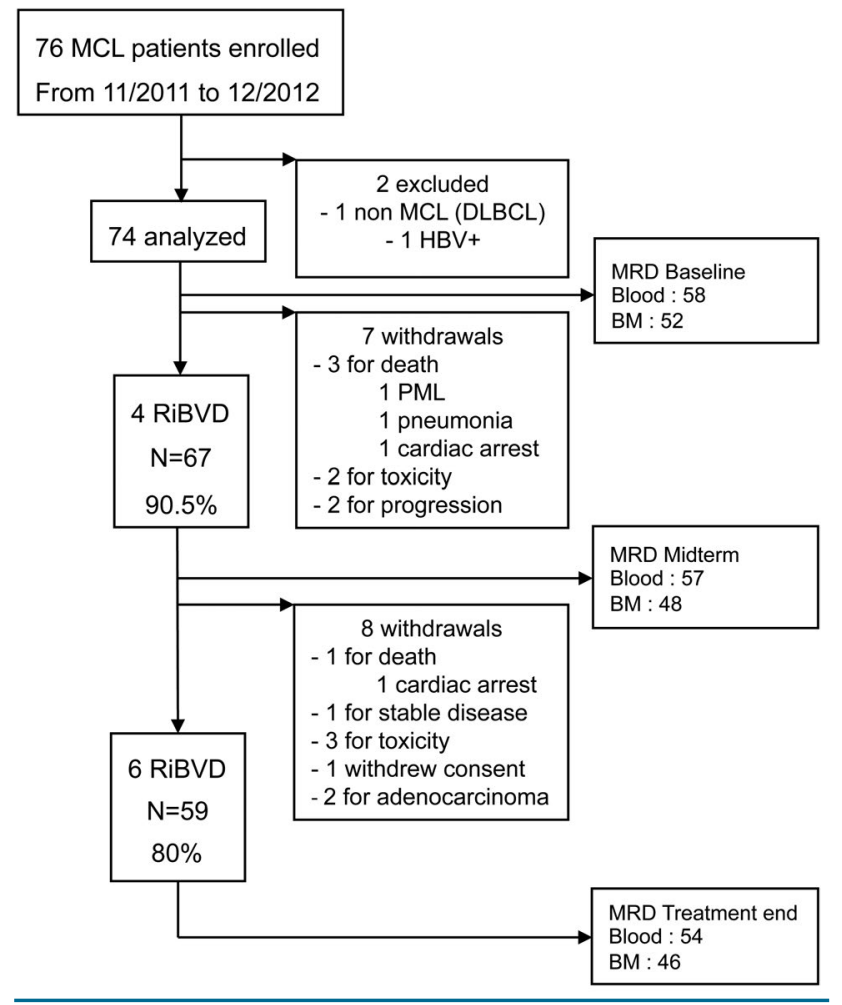

Figure 1. Consort diagram for the RiBVD phase 2 trial. MCL: mantle cell lymphoma; DLBCL: diffuse large B-cell lymphoma; HBV: hepatitis B virus; MRD: minimal residual disease; BM: bone marrow; PML: progressive multifocal leukoencephalopathy.

therapy. Two patients were excluded - one because of a misdiagnosis of MCL (diffuse large B-cell lymphoma) and one because of exclusion criteria (hepatitis B) - leaving 74 patients for data analyses (Figure 1). Seventy-one patients had MCL confirmed by central review. The diagnosis was made on tumor biopsies (45 on lymph nodes and 26 on extra-nodal tissue). Due to unsuccessful tissue biopsy in three patients, a diagnosis of MCL was made by flow cytometry in peripheral blood (1 patient) or bone marrow ( 2 patients). Ki67 staining was performed in 56 patients, and was found $\geq 30 \%$ positive in $59 \%$ of these patients (31 of 56 patients) (Table 2).

\section{Treatment response}

Seventy-four patients initiated therapy. Sixty-seven patients received at least four cycles $(90.5 \%)$ of treatment

Table 1. Patients' demographics and clinical characteristics.

Characteristics $\mathrm{N}$. $\%$

Age (years)
median
range

\begin{tabular}{lll} 
Sex & & \\
male & 49 & 66 \\
female & 25 & 34 \\
\hline
\end{tabular}

WHO Performance Status

$\begin{array}{lll}0-1 & 73 & 85 \\ 2-4 & 11 & 15\end{array}$

Lactate dehydrogenase

$\begin{array}{lll}\text { normal } & 44 & 61 \\ >\text { normal } & 28 & 39\end{array}$

B symptoms

$\begin{array}{lll}\text { no } & 56 & 76\end{array}$

$\begin{array}{lll}\text { yes } & 17 & 24\end{array}$

Ann Arbor stage

II

III-IV

4 70

Bulky tumor

$\begin{array}{lll}\text { no } & 52 & 71 \\ \text { yes } & 21 & 29\end{array}$

yes 21

\begin{tabular}{lcc} 
Extranodal involvement & & \\
no & 7 & 9 \\
yes & 67 & 91 \\
\hline
\end{tabular}

Bone marrow involvement

$\begin{array}{lll}\text { no } & 24 & 34 \\ \text { yes } & 46 & 66\end{array}$

$\begin{array}{lll}\text { Spleen involvement } & & \\ \text { no } & 38 & 52 \\ \text { yes } & 35 & 48\end{array}$

\begin{tabular}{lcc} 
yes & 35 & 48 \\
\hline MIPI score & & \\
$\quad$ low & 2 & 3 \\
intermediate & 12 & 17 \\
$\quad$ high & 58 & 80 \\
MIB1 / ki67 prolferation index & & \\
$\quad<30 \%$ & 21 & 41 \\
$\geq 30 \%$ & 30 & 59 \\
\hline
\end{tabular}

Pathology

$\begin{array}{lll}\text { classic } & 61 & 86 \\ \text { blastoid } & 10 & 14\end{array}$

$\begin{array}{lll}\text { blastoid } & 10 & 14\end{array}$

WHO:World Heath Organization; MIPI: Mantle Cell Lymphoma International Prognostic Index. 
and $59(80 \%)$ received all six planned cycles (Figure 1). Of the planned 444 cycles, $406(91.5 \%)$ were administered. Fifteen patients stopped therapy before receiving all six cycles (Figure 1). After four cycles, the overall response rate was $86.5 \%$ (64/74) and the complete response rate (confirmed and unconfirmed complete responses) was $56.5 \%$ (42/74). At the end of treatment, the overall response rate was $84 \%(62 / 74)$ and the complete response rate was $75.5 \%$ (56/74). FDG-PET evaluations were performed after four cycles of treatment in 64 patients $(100 \%$ of the 64 responders) and after cycle 6 in 59 patients ( $95 \%$ of the 62 responders). Interim and final FDG-PET were negative in $64 \%(41 / 64)$ and in $78 \%$ of evaluated patients (46/59), respectively.

\section{Molecular minimal residual disease in blood and bone marrow}

Molecular MRD was assessed in a total of 58 of the 74 patients eligible for MRD analysis (in all, 732 samples were assessed, see Online Supplementary Figure S1). Molecular MRD analysis was not possible in 16 of 74 MRD-eligible patients because of a lack of MRD target $(n=6)$, missing follow-up samples $(n=9)$ or because an MRD target reference sample was not available $(\mathrm{n}=1)$ (Online Supplementary Figure S1). After four cycles (midterm), 57 patients were analyzed for molecular MRD (57 peripheral blood samples; 48 bone marrow samples, of which 48 patients with paired bone marrow and peripheral blood MRD samples, at the mid-term analysis). Of these, 50 patients were negative for molecular MRD (32 in complete remission, 18 in partial remission) and seven were positive ( 2 in complete remission, 4 in partial remission and 1 with stable disease) in the blood and/or bone marrow, for a molecular response rate of $79 \%$ (defined by a quantitative polymerase chain reaction assay with a sensitivity of $\left.10^{-5}\right)$. After six cycles of treatment, 54 patients were analyzed for molecular MRD (54 peripheral blood samples; 46 bone marrow samples, of which 46 patients with paired bone marrow and peripheral blood samples for MRD analysis at the end of treatment). Of these 54 patients, 41 were MRD-negative (39 in complete remis- sion, 1 in partial remission and 1 with stable disease) and 13 were MRD-positive (8 in complete remission, 4 in partial remission and 1 with progressive disease) in blood and/or bone marrow ( $76 \%$ molecular response rate) (Figure 2 and Online Supplementary Figure S1).

Molecular MRD response rates were then analyzed separately in the peripheral blood versus bone marrow at the mid-term follow-up time-point (after 4 treatment cycles) and at the end of treatment (after 6 cycles). Blood samples were molecular MRD-negative from 88\% (50/57) of patients after four cycles and 87\% (47/54 patients) after six cycles (Figure 2C, left panel). The corresponding percentages for bone marrow samples were $77 \%$ (37/48 patients) after four cycles of treatment and 76\% (35/46 patients) at the end of treatment (after 6 cycles) (Online Supplementary Figure S3).

\section{Survival analyses and prognostic factors}

With a median follow-up time of 52 months, 74 patients were evaluable. Overall, 24 patients died, four during treatment (2 from cardiac arrest, 1 with pneumonia and 1 with progressive multifocal leukoencephalopathy, after cycle 3) and 20 during follow-up (16 due to progressive disease, 1 from pancreatic adenocarcinoma, 1 from cardiac arrest and 2 from unknown causes).

The 2-year PFS was $70.3 \%$ compared to $57.6 \%$ at 4 years. The 2 -year OS was $81.1 \%$ compared to $71.3 \%$ at 4 years. The Mantle Cell Lymphoma International Prognostic Index (MIPI) score was not predictive for PFS or OS perhaps due to the small number of patients because a trend could be observed (Table 2 and Online Supplementary Figure S2A). Indeed, the 4-year OS for the 58 MIPI high-risk patients was $66.8 \%$ compared to $85.7 \%$ for the $14 \mathrm{MIPI}$ low- or intermediate-risk patients $(P=0.13)$. Neither histology (classical subtype versus blastoid subtype) nor Ki67 expression ( $<30 \%$ versus $\geq 30 \%$ of positive MCL cells in the tumor biopsy) was predictive for OS ( $P=0.10$ and $P=0.24$, respectively) or PFS ( $P=0.08$ and $P=0.13$, respectively) (Table 2 and Online Supplementary Figure S2B). Clinical responses (complete or partial response versus no response), as assessed by the Cheson 1999 criteria, were

Table 2. Prognostic factors for progression-free survival and overall survival.

\begin{tabular}{lccc}
\hline Prognostic factors & N. & P for PFS & P for OS \\
Pathology (classic vs. blastoid form) & 71 & 0.08 & 0.10 \\
MIPI score (high vs. low/Intermediate) & 72 & 0.18 & 0.13 \\
\hline Ki67 (< vs. $\geq 30 \%)$ & 51 & 0.35 & 0.24 \\
Response IWC 1999 (CR vs. PR vs. failure) & 74 & $<0.0001$ & $<0.0001$ \\
\hline FDG-PET midterm & 64 & 0.19 & 0.57 \\
FDG-PET treatment end. & 59 & 0.48 & 0.98 \\
\hline MRD blood and/or bone marrow at mid-term (neg 45; pos 12) & 57 & 0.20 & 0.33 \\
MRD blood and/or bone marrow at treatment end (neg 41; pos 13) & 54 & 0.04 & 0.02 \\
\hline MRD blood mid-term (neg 50; pos 7) & 57 & 0.01 & 0.047 \\
MRD blood treatment end (neg 47; pos 7) & 54 & $<0.0001$ & $<0.0001$ \\
\hline MRD bone marrow midterm (neg 37; pos 11) & 48 & 0.24 & 0.41 \\
MRD bone marrow treatment end (neg 35; pos 11) & 46 & 0.20 & 0.19
\end{tabular}

N: number of patients who could be evaluated ; MIPI score,: Mantle-Cell Lymphoma International Prognosis Index; Ki67/Mib1, proliferation index score; Response IWC 1999, response according to the 1999 International Workshop Criteria; PF: progression free survival; OS: overall survival (at 4 years), respectively; CR: complete response; PR: partial response; FDG-PET, [18F] fluorodeoxyglucose positron emission tomography; mid-term, analysis after four cycles; treatment end, analysis after six cycles; MRD, (molecular) minimal residual disease. 
highly predictive for PFS or OS whether determined at the mid-term staging or at the end of treatment. There were no survival differences (PFS or OS) between patients in partial or complete remission. Neither mid-term nor final FDGPET scan responses were predictive for PFS or OS. The most highly predictive factor for PFS and OS $(P<0.0001)$ was MRD status in peripheral blood at the end of treatment (Figure 2C, right panel and Table 2). Molecular blood MRD status at mid-term was also significant for PFS $(P=0.01)$ and weakly significant for OS $(P=0.047)$ (Table 2$)$. By contrast, MRD status in the bone marrow after four cycles of treatment (mid-term) or at the end of treatment was not predictive for either PFS or OS (see Online Supplementary Figure S3 for end-of-treatment data). The 4year OS for patients who were MRD-negative in blood at the end of treatment $(n=47 / 54)$ was $86.6 \%$ compared to $28.6 \%$ for blood MRD-positive patients $(n=7 / 54)$.

Continued molecular remission status in the peripheral blood after therapy (at the 12-month follow-up) was significantly associated with longer PFS (33 patients; 4-year PFS $97 \%)$. By contrast, the median PFS for patients who remained MRD-positive $(n=6)$ or who had converted to an MRD-positive status in the peripheral blood by the 12month follow-up ( $\mathrm{n}=7$ ) was 11 and 26 months, respectively.
A

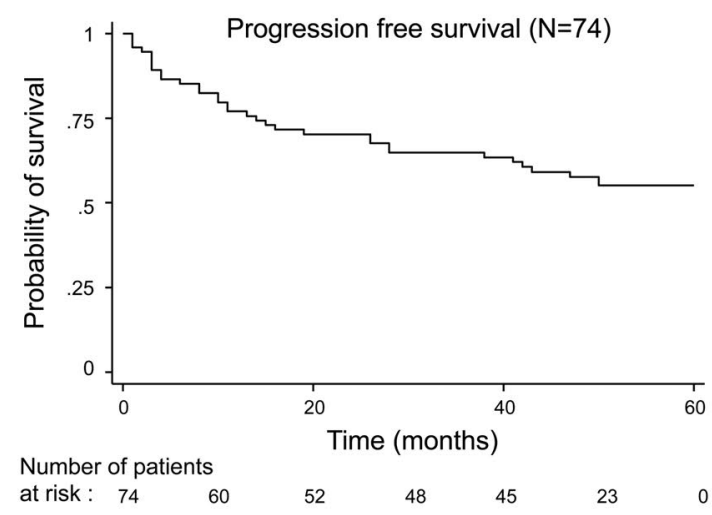

B

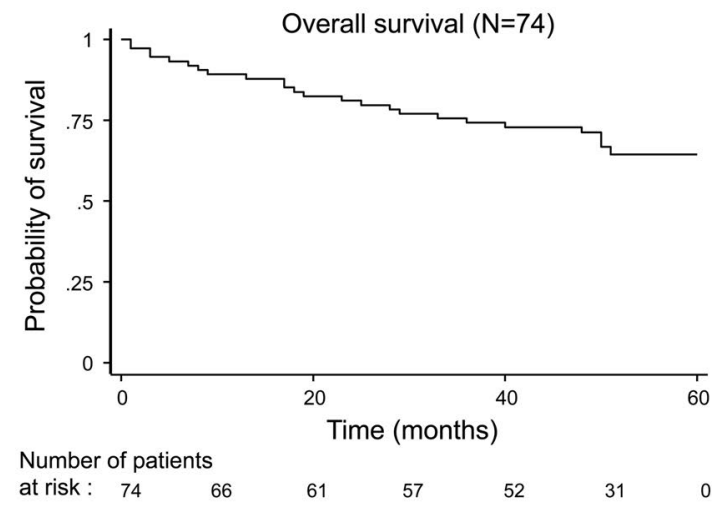

C
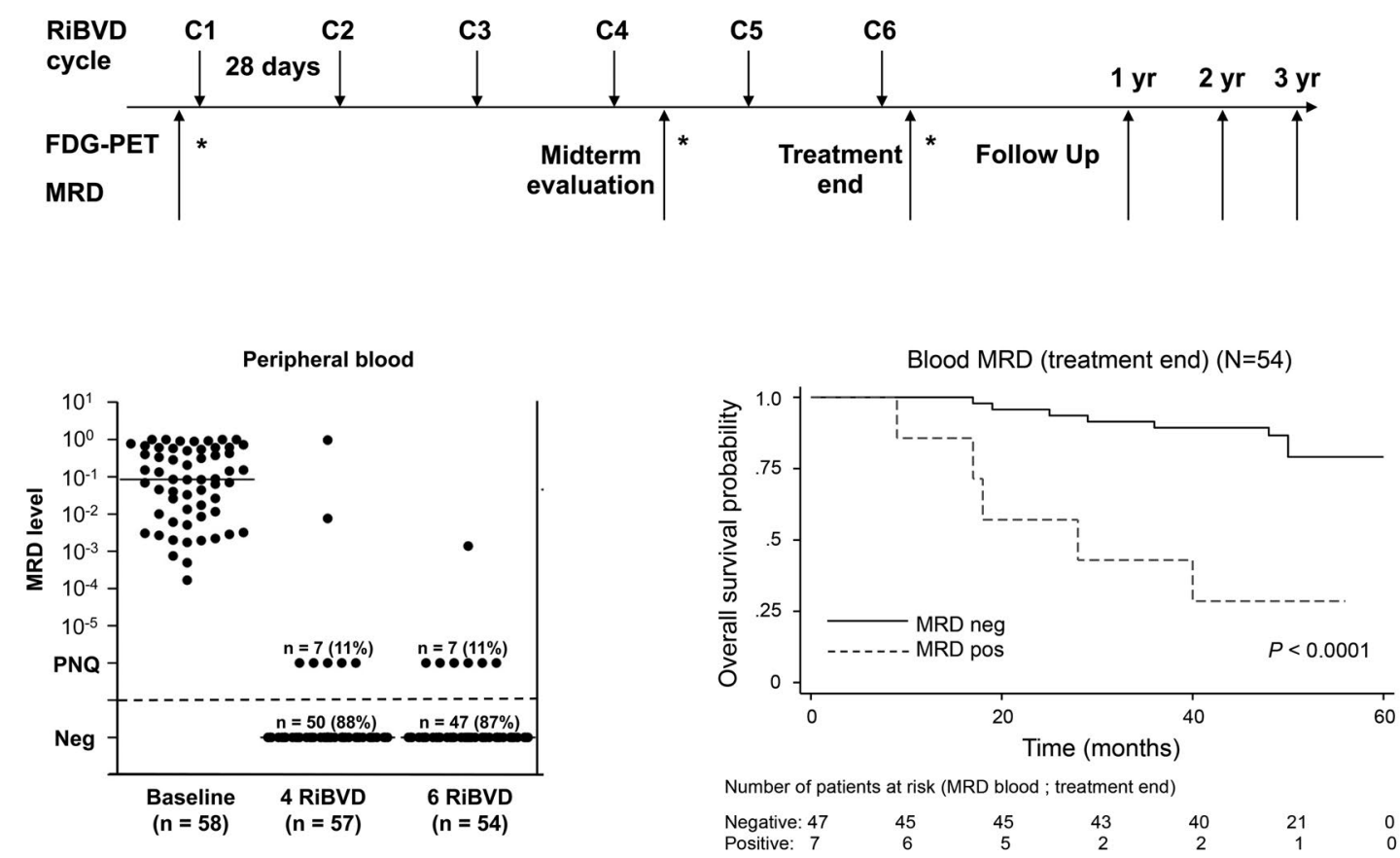

Figure 2. Survival of patients with mantle cell lymphoma following frontline treatment with the RiBVD regimen. (A) Progression-free survival of the 74 patients. (B) Overall survival of the 74 patients (C) Molecular response rates and overall survival according to molecular residual disease (MRD) status in peripheral blood after six cycles of RiBVD (treatment end). 


\section{Toxicity}

Fifteen patients of $74(20 \%)$ stopped treatment before the sixth cycle, four because of death (1 case each of pneumonia and progressive multifocal leukoencephalopathy and 2 cardiac arrests), five because of grade 3-4 toxicity [septicemia $(n=1)$, neuropathy $(n=2)$, digestive tract toxicity $(n=1)$ and pleural effusion $(n=1)]$, three because of progression or stable disease and three for other causes (Figure 1). During treatment, 49 of 74 patients $(66.2 \%)$ developed grade 3-4 hematologic toxicities $(51 \%$ neutropenia, $35 \%$ thrombocytopenia and 19\% anemia) (Table 3). Neutropenia translated into febrile neutropenia in 11 patients $(11 \%)$, which was grade 3-4 in six. Lymphopenia at the end of treatment was reported in $65 \%$ of the patients (48/70) and was mainly grade 3-4 (lymphocytes $<0.5 \times 10^{9} / \mathrm{L}$ ) (Table 3). Persistent grade 3-4 lymphopenia was seen in $28.8 \%$ of patients at 1 year after the completion of treatment (17 of 59 surviving patients who could be evaluated). Forty-two patients $(56.7 \%)$ had non-hematologic grade 3-4 toxicities at the end of treatment (Table 3 ). The most frequent non-hematologic toxicities (seen in more than $10 \%$ of patients) were fatigue, peripheral neuropathy and fever with or without neutropenia, which occurred in $18.5 \%(n=14), 15 \%(n=11)$ and $15(n=11)$ of cases, respectively (Table 3 ). Other toxicities were reported in four or fewer patients (i.e. less than 6\%) and were as follows: pulmonary toxicity, cardiac toxicity, hyperglycemia, elevated transaminases, digestive tract toxicity, cutaneous rash, allergy and fever without neutropenia. No patient experienced cytomegalovirus reactivation or pneumocystis infection.
Twenty-four episodes of infection were declared as serious adverse events. These represented one-third of the 76 serious adverse events reported during the 406 cycles of therapy, or during follow up. They included seven cases of opportunistic infection (4 cases during treatment and 3 further cases 1 year after the end of treatment), which were as follows; herpes zoster $(n=3)$, progressive multifocal leukoencephalopathy $(n=1)$, cytomegalovirus colitis $(n=1)$, listeriosis $(n=1)$ and oral candidosis $(n=1)$. Additional infections were pneumonia $(n=9)$, staphylococcal infection $(n=2)$, followed by non-recurring infections of various types (no more than 1 case each, as follows; acute pyelonephritis, bronchitis, catheter site infection, upper aero-digestive tract infection and Clostridium difficileinduced colitis).

Regarding neurotoxicity, grade 2 to 4 neuropathy was observed in $21.5 \%$ of patients (16 of 74 patients) (Table 3 ). Neuropathy was generally reported after cycle 3 of treatment. Bortezomib was stopped indefinitely in ten of the 11 patients with grade 3-4 neurotoxicity but not in cases of grade 2 toxicity. Partial reversibility of neuropathy was reported in 13 of the 16 patients (81\%) with grade 2 to 4 neurotoxicity.

\section{Discussion}

We report the results of a prospective, phase II study by the French LYSA group. The study aimed to test the efficacy of six cycles of RiBVD, without maintenance therapy, for first-line treatment of MCL patients aged $\geq 65$

Table 3. Hematologic and non-hematologic toxicity.

\begin{tabular}{|c|c|c|c|c|c|c|}
\hline \multirow[b]{2}{*}{ Events } & \multicolumn{2}{|c|}{ All Grades } & \multicolumn{2}{|c|}{ Grade 3} & \multicolumn{2}{|c|}{ Grade 4} \\
\hline & N. & $\%$ & N. & $\%$ & N. & $\%$ \\
\hline Neutropenia & 52 & 70 & 17 & 23 & 21 & 28.5 \\
\hline Thrombocytopenia & 67 & 90.5 & 18 & 24 & 8 & 11 \\
\hline Lymphopenia & 67 & 95.7 & 38 & 54 & 8 & 11 \\
\hline Anemia & 70 & 94.5 & 6 & 8 & 0 & 0 \\
\hline Fatigue & 56 & 75.5 & 12 & 16 & 2 & 2.5 \\
\hline Neuropathy & 32 & $43^{*}$ & 10 & 13.5 & 1 & 1.5 \\
\hline Fever & 35 & 47 & 5 & 6.5 & 2 & 2.5 \\
\hline Febrile neutropenia & 8 & 11 & 4 & 5.5 & 2 & 2.5 \\
\hline Lung & 26 & 35 & 4 & 5.5 & 2 & 2.5 \\
\hline Cardiac & 16 & 21.5 & 4 & 5.5 & 1 & 1.5 \\
\hline Hyperglycemia & 23 & 31 & 4 & 5.5 & 0 & 0 \\
\hline Rash & 25 & 34 & 3 & 4 & 0 & 0 \\
\hline GOT/GPT & 29 & 39 & 3 & 4 & 0 & 0 \\
\hline Digestive tract & 35 & 47 & 3 & 4 & 0 & 0 \\
\hline Allergy & 24 & 32 & 1 & 1 & 0 & 0 \\
\hline Weight loss & 25 & 34 & 0 & 0 & 0 & 0 \\
\hline Nausea & 25 & 34 & 0 & 0 & 0 & 0 \\
\hline Bilirubin & 12 & 16 & 0 & 0 & 0 & 0 \\
\hline Creatinine & 24 & 32.5 & 0 & 0 & 0 & 0 \\
\hline Ear & 2 & 3 & 0 & 0 & 0 & 0 \\
\hline Infusion-related reaction & 18 & 24 & 0 & 0 & 0 & 0 \\
\hline Calcium & 4 & 5 & 0 & 0 & 0 & 0 \\
\hline
\end{tabular}

GOT/GPT: glutamic oxaloacetic transaminase/glutamic-pyruvic transaminase; CMV: cytomegalovirus. *includes grade $2=6.8 \%$ (n=5/74). 
years. With a median follow up of 52 months, the 2-year PFS of the 74 patients with analyzable data was $70 \%$, thus reaching the primary objective of the study which was to improve median PFS by 6 months compared to the reported 18-month PFS for patients treated with R-CHOP., ${ }^{3,4}$ The 4-year PFS (57.6\%) observed here for RiBVD-treated patients is in line with the median PFS reported for the BR regimen (35.4 months) and other bortezomib-containing regimens such as 24.7 months for VR-CAP (bortezomib, rituximab, cyclophosphamide, doxorubicin, and prednisone) and 26 months for the $\mathrm{RiPAD}+\mathrm{C}$ regimen (rituximab, bortezomib, doxorubicin, dexamethasone, and chlorambucil). ${ }^{7-9}$ The favorable PFS with the RiBVD regimen may be related to the marked depth of response $(75.7 \%$ rate of confirmed and unconfirmed complete responses according to the Cheson 1999 criteria; 78\% rate of complete responses according to the Cheson 2007 criteria). Although not strictly comparable outside of a randomized trial, it is worth noting that complete response rates with other regimens are lower: R-CHOP (34\%), BR ( $40 \%)$, VR-CAP $(53 \%)$ and RiPAD+C (51\%). ${ }^{2,3,7,8}$ The higher rate of complete responses (confirmed and unconfirmed) with the RiBVD regimen translated into higher molecular response rates $[76 \%$ of patients $(41 / 54)$ were $\mathrm{MRD}$-negative in blood and/or bone marrow at the end of treatment] compared to published data for R-CHOP in older MCL patients [67\% MRD negativity (54/81 patients)]. ${ }^{20}$ It is worth noting that $80 \%$ of patients in our study had high-risk MIPI scores and that $59 \%$ had $\geq 30 \%$ Ki67 positivity (range, $5 \%$ to $95 \%$ positivity) which appears high compared to the percentages in other studies in MCL patients over 65 years old (see Online Supplementary Table S4).

In keeping with results of high-dose cytarabine treatment in younger MCL patients, ${ }^{21-23}$ a recent phase II trial from an Italian group (FIL) confirmed the efficacy of the RBAC500 regimen (which associates rituximab, bendamustine and cytarabine) for treatment of older MCL patients. ${ }^{12}$ In updated clinical results for the R-BAC500 regimen, complete response rates of $93 \%$ and molecular response rates of $78 \%(35 / 45)$ in the blood have been reported. ${ }^{24}$ The 2 -year PFS and OS were estimated as $83 \%$ and $86 \%$, respectively. ${ }^{24}$ However, it is worth noting that the baseline characteristics of the MCL cohort treated with RBAC500, the patients in our study and those in other published series of MCL cases differ quite widely (Online Supplementary Table S4).

The rate of treatment discontinuation in our study was $20 \%$ (15/74 patients). This is broadly in line with rates reported for R-CHOP [17\% (43/242)] and VR-CAP [18.8\% $(45 / 240)]$ in older MCL patients treated in first line, ${ }^{7}$ and is lower than that reported with the R-BAC500 regimen $(33 \%)^{24}$ (Online Supplementary Table S4). The reported rates of premature therapy cessation for the BR and R-CHOP regimens are $8 \%$ and $5 \%$, respectively. ${ }^{10}$

The $5.4 \%$ rate of toxic deaths reported here with the RiBVD regimen is in line with that observed for other first-line regimens used in older MCL patients. For instance, in a phase III study comparing the R-CHOP regimen to VR-CAP, Robak and colleagues reported 14/242 deaths $(6 \%)$ and $11 / 240$ deaths $(5 \%)$, in the R-CHOP and VR-CAP arms, respectively. ${ }^{7}$ Of these deaths, a total of six were due to infection and three to cardiac failure.

A trial of lenalidomide, bendamustine and rituximab recently documented a complete response rate of $64 \%$, with molecular MRD negativity reached in 34\% patients. Toxicity, however, was greater than with the RiBVD regimen, with infectious grade 3-5 toxicities seen in $42 \%$ of the 51 recruited patients. ${ }^{13}$ Two large phase III trials of BR \pm ibrutinib or BR \pm ACP196 (acalabrutinib) are still ongoing and results are pending.

Grade 3-4 hematologic toxicities observed with the RiBVD regimen (51\% neutropenia and 35\% thrombocytopenia) were in line with those seen in patients treated with other regimens such as R-CHOP (60\% neutropenia and $18 \%$ thrombocytopenia), VR-CAP (85\% and $57 \%$, respectively) or R-BAC (rituximab, bendamustine and cytarabine; $49 \%$ and $52 \%$, respectively). ${ }^{2,7,24}$

Lymphopenia (65\% of grade $3-4$ ), which is known to occur with the $\mathrm{BR}$ regimen, may contribute, with neutropenia, to the relatively high number of infectious episodes seen in this study., The rate of lymphopenia at 1 year was $32.5 \%$, which is indicative of longer-term immunosuppression with the RiBVD regimen. Whether this is related to the use of dexamethasone or to the immunosuppressive effects of bendamustine remains to be established but indicates that precautionary measures to control infection are advisable.

Contrary to published results for subcutaneous administration of bortezomib, we noted a relatively high incidence of grade 3-4 neurotoxicity, which is a limiting factor for the RiBVD regimen. ${ }^{25}$ Although the incidence was comparable to that observed in our previous $\mathrm{RiPAD}+\mathrm{C}$ trial (18\% grade 3-4 toxicity, $7 / 39$ patients), in which bortezomib was administered intravenously at the same dose, ${ }^{8}$ it is higher than that observed in other studies using comparable intravenous doses in which grade 3-4 toxicity was reported in $7 \%$ to $8 \%$ of patients treated with R-BV (rituximab, bendamustine, and bortezomib) and VR-CAP regimens.,11 Further investigations will be required to understand the reason for this. Of note in this respect is the discovery of genetic risk loci for severe peripheral neuropathy in European patients with multiple myeloma treated with bortezomib. ${ }^{26}$

In this study, molecular response in peripheral blood at the end of treatment (after 6 RiBVD cycles) was identified as a major predictive factor for PFS and OS, thus further emphasizing the importance of the depth of response, beyond standard clinical complete response, in $\mathrm{MCL}^{20}$ Indeed, there was no difference in OS between patients in complete or partial remission at the end of treatment, as defined by the IWG criteria with or without FDG-PET. This finding supports the notion that PET and molecular MRD provide different prognostic information in MCL, probably because they are measuring different types of disease activity, in different disease compartments, with differing sensitivities. The maximum standardized uptake value (SUVmax) defined by FDG-PET, also described as an independent prognosis factor, was not analyzed in our cohort. ${ }^{27}$

Unexpectedly, neither the MIPI nor Ki67 scores (30\% cut-off) had any impact on PFS or OS with the RiBVD regimen. This may reflect differences in treatment efficacy by RiBVD in patients with high-risk MIPI scores $70 \%$ OS at 36 months), compared to the efficacy of historical treatment controls in the original patient cohort that was used to define the high-risk MIPI score $(40 \%$ OS at 36 months).$^{28}$

Peripheral blood, but not bone marrow-based MRD status, was highly predictive of PFS and OS in this study (4-year OS of $86.6 \%$ for MRD-negative patients com- 
pared to $28.6 \%$ for MRD-positive patients; $(P<0.0001)$. While for peripheral blood this is broadly in keeping with findings of the EU-MCL network for other treatment regimens,$^{20}$ results concerning the prognostic impact of bone marrow molecular MRD, in patients treated with RiBVD, differ. ${ }^{20}$ The prognostic impact of MRD in patients treated with the R-BAC500 regimen has not been reported as yet. $^{24}$ One avenue of investigation for clarification of these issues will be testing of 'next generation' cellular and molecular methods of MRD detection. Multi-parametric flow cytometry, although requiring very high levels of expertise, has been shown to be feasible and provide satisfactory sensitivity, when compared to highly standardized quantitative polymerase chain reaction methods in MCL. ${ }^{29}$ For molecular MRD, droplet digital polymerase chain reaction analysis is gaining interest ${ }^{30}$ as is molecular MRD assessment in circulating cell-free DNA in B-cell non-Hodgkin lymphoma. ${ }^{31-33}$ Combinatorial approaches (metabolic, cellular/molecular) as reported here for MCL, and in follicular lymphoma, ${ }^{34}$ will also be useful. Ultimately, careful investigation of residual disease, particularly by combinatorial approaches, will be needed to further refine $\mathrm{MRD}$-driven precision medicine approaches in lymphoma, and other cancers..$^{35}$

$M R D$ positivity at the end of treatment or at 1 year of follow-up was found to be highly predictive for early relapse (at 11 and 26 months, respectively) in patients treated with the RiBVD regimen. Although numbers of $M R D$-negative patients were small, these findings add further weight to the notion that achieving durable molecular remission is an important goal in MCL. Indeed, maintenance therapy and/or pre-emptive treatment directed to patients in molecular relapse or remaining MRD-positive after treatment has been shown to play a significant role in prolonging clinical response in MCL. ${ }^{2,23,36,37}$ The choice of maintenance or a pre-emptive therapy strategy may depend on the nature of initial therapy, as highlighted by a recent study that failed to show the benefit of rituximab maintenance after bendamustine. ${ }^{38}$

In conclusion, our results identify the combination of rituximab, bendamustine bortezomib and dexamethasone, without maintenance therapy, as a promising treatment option in MCL patients $\geq 65$ years old. The RiBVD regimen compares favorably with other treatment strategies used in this setting, although randomized trials are still lacking. Prolonged PFS appears to result from rapid clearance of (re)circulating tumor B cells in the post-induction phase. Continued molecular remission in the blood was predictive of prolonged survival, indicating that molecular MRD monitoring and molecular response offer significant potential as precision medicine tools for early and late clinical decision-making in MCL.

\section{Acknowledgments}

We thank Dr Jean Marie Quésada, biostatistician at the Grenoble Clinical Investigation Center (INSERM CIC 1406), for his help in data analysis, Valérie Rolland-Neyret and Roseline Delepine, data coordinators of the trial, for their excellent work, and all of the clinicians, not mentioned in the author list, who enrolled patients. We also thank the hematopathologists of the LYSA Pathology Commission for their pathology review (Dr. Danielle Canioni, Dr. Barbara Burroni, Prof. Alexandra TraverseGlehen, Prof. Antoine Martin) and staff of the LYSA-Pathology core facility for providing their expert assistance for immunohistochemistry. We thank Estelle Gimenez for excellent technical coordination of the MRD work. We also thank our partners Mundipharma, Jannsen-Cilag, and Roche for funding. MBC acknowledges additional institutional support from the Universite Grenoble-Alpes, Université Bourgogne-Franche Comté, INSERM and CNRS. Research funding via the French National Cancer Institute and the ITMO Cancer 'Epigenetics and Cancer programme' is also acknowledged. Finally, we thank Professor Andre Goy, chairman and executive director of the John Theurer Cancer Center, Hackensack for his critical review of the manuscript.

\section{References}

1. Campo E, Rule S. Mantle cell lymphoma: evolving management strategies. Blood. 2015;125(1):48-55

2. Kluin-Nelemans HC, Hoster E, Hermine O, et al. Treatment of older patients with mantle-cell lymphoma. $N$ Engl J Med. 2012;367(6):520-531

3. Lenz G, Dreyling $M$, Hoster $E$, et al. Immunochemotherapy with rituximab and cyclophosphamide, doxorubicin, vincristine, and prednisone significantly improves response and time to treatment failure, but not long-term outcome in patients with previously untreated mantle cell lymphoma: results of a prospective randomized trial of the German Low Grade Lymphoma Study Group (GLSG). J Clin Oncol. 2005;23(9):1984-1992.

4. Howard OM, Gribben JG, Neuberg DS, et al. Rituximab and CHOP induction therapy for newly diagnosed mantle-cell lymphoma: molecular complete responses are not predictive of progression-free survival. J Clin Oncol. 2002;20(5):1288-1294.

5. Baumann U, Fernandez-Saiz V, Rudelius M, et al. Disruption of the PRKCD-FBXO25-
HAX-1 axis attenuates the apoptotic response and drives lymphomagenesis. Nat Med. 2014;20(12):1401-1409.

6. Goy A, Bernstein SH, Kahl BS, et al. Bortezomib in patients with relapsed or refractory mantle cell lymphoma: updated time-to-event analyses of the multicenter phase 2 PINNACLE study. Ann Oncol. 2009;20(3):520-525.

7. Robak T, Huang H, Jin J, et al. Bortezomibbased therapy for newly diagnosed mantlecell lymphoma. N Engl J Med. 2015;372(10):944-953.

8. Houot R, Le Gouill S, Ojeda Uribe M, et al. Combination of rituximab, bortezomib, doxorubicin, dexamethasone and chlorambucil $(\mathrm{RiPAD}+\mathrm{C})$ as first-line therapy for elderly mantle cell lymphoma patients: results of a phase II trial from the GOELAMS. Ann Oncol. 2012;23(6):1555-1561.

9. Rummel MJ, Niederle N, Maschmeyer G, et al. Bendamustine plus rituximab versus CHOP plus rituximab as first-line treatment for patients with indolent and mantle-cell lymphomas: an open-label, multicentre, randomised, phase 3 non-inferiority trial. Lancet. 2013;381(9873):1203-1210.

10. Flinn IW, van der Jagt R, Kahl BS, et al. Randomized trial of bendamustine-ritux- imab or R-CHOP/R-CVP in first-line treatment of indolent NHL or MCL: the BRIGHT study. Blood. 2014;123(19):29442952

11. Friedberg JW, Vose JM, Kelly JL, et al. The combination of bendamustine, bortezomib, and rituximab for patients with relapsed/refractory indolent and mantle cell non-Hodgkin lymphoma. Blood. 2011;117 (10):2807-2812.

12. Visco C, Finotto S, Zambello R, et al Combination of rituximab, bendamustine, and cytarabine for patients with mantle-cell non-Hodgkin lymphoma ineligible for intensive regimens or autologous transplantation. J Clin Oncol. 2013;31(11):14421449.

13. Albertsson-Lindblad A, Kolstad A, Laurell A, et al. Lenalidomide-bendamustine-rituximab in patients older than 65 years with untreated mantle cell lymphoma. Blood. 2016;128(14):1814-1820.

14. Rummel MJ, Al-Batran SE, Kim SZ, et al Bendamustine plus rituximab is effective and has a favorable toxicity profile in the treatment of mantle cell and low-grade non-Hodgkin's lymphoma. J Clin Oncol. 2005;23(15):3383-3389.

15. Robinson KS, Williams ME, van der Jagt 
RH, et al. Phase II multicenter study of bendamustine plus rituximab in patients with relapsed indolent $\mathrm{B}$-cell and mantle cell non-Hodgkin's lymphoma. J Clin Oncol. 2008;26(27):4473-4479.

16. Klapper W, Hoster E, Determann O, et al. Ki-67 as a prognostic marker in mantle cell lymphoma-consensus guidelines of the pathology panel of the European MCL Network. J Hematop. 2009;2(2):103-111.

17. Meignan M, Gallamini A, Haioun C. Report on the First International Workshop on Interim-PET-Scan in Lymphoma. Leuk Lymphoma. 2009;50(8):1257-1260.

18. Gimenez E, Chauvet M, Rabin L, et al. Cloned IGH VDJ targets as tools for personalized minimal residual disease monitoring in mature lymphoid malignancies; a feasibility study in mantle cell lymphoma by the Groupe Ouest Est d'Etude des Leucemies et Autres Maladies du Sang. Br J Haematol. 2012;158(2):186-197.

19. Pott C, Schrader C, Gesk S, et al. Quantitative assessment of molecular remission after high-dose therapy with autologous stem cell transplantation predicts long-term remission in mantle cell lymphoma. Blood. 2006;107(6):2271-2278.

20. Pott C, Hoster E, Delfau-Larue MH, et al. Molecular remission is an independent predictor of clinical outcome in patients with mantle cell lymphoma after combined immunochemotherapy: a European MCL intergroup study. Blood. 2010;115(16): 3215-3223

21. Geisler CH, Kolstad A, Laurell A, et al. Nordic MCL2 trial update: six-year followup after intensive immunochemotherapy for untreated mantle cell lymphoma followed by BEAM or BEAC + autologous stem-cell support: still very long survival but late relapses do occur. Br J Haematol. 2012;158(3):355-362

22. Hermine O, Hoster E, Walewski J, et al. Addition of high-dose cytarabine to immunochemotherapy before autologous stem-cell transplantation in patients aged
65 years or younger with mantle cell lymphoma (MCL younger): a randomised, open-label, phase 3 trial of the European Mantle Cell Lymphoma Network. Lancet. 2016;388(10044):565-575.

23. Le Gouill S, Thieblemont C, Oberic L, et al. Rituximab after autologous stem-cell transplantation in mantle-cell lymphoma. N Engl J Med. 2017;377(13):1250-1260.

24. Visco C, Chiappella A, Nassi L, et al Rituximab, bendamustine, and low-dose cytarabine as induction therapy in elderly patients with mantle cell lymphoma: a multicentre, phase 2 trial from Fondazione Italiana Linfomi. Lancet Haematol. 2017;4(1):e15-e23.

25. Moreau P, Pylypenko H, Grosicki S, et al Subcutaneous versus intravenous administration of bortezomib in patients with relapsed multiple myeloma: a randomised, phase 3 , non-inferiority study. Lancet Oncol. 2011;12(5):431-440.

26. Magrangeas F, Kuiper R, Avet-Loiseau H, et al. A genome-wide association study identifies a novel locus for bortezomib-induced peripheral neuropathy in european patients with multiple myeloma. Clin Cancer Res. 2016;22(17):4350-4355.

27. Bodet-Milin C, Touzeau C, Leux C, et al Prognostic impact of 18F-fluoro-deoxyglucose positron emission tomography in untreated mantle cell lymphoma: a retrospective study from the GOELAMS group. Eur J Nucl Med Mol Imaging. 2010;37(9): 1633-1642

28. Hoster E, Dreyling M, Klapper W, et al. A new prognostic index (MIPI) for patients with advanced-stage mantle cell lymphoma. Blood. 2008;111(2):558-565.

29. Cheminant $M$, Derrieux $C$, Touzart $A$, et al. Minimal residual disease monitoring by 8 color flow cytometry in mantle cell lymphoma: an EU-MCL and LYSA study. Haematologica. 2016;101(3):336-345

30. Drandi D, Ferrero S, Ladetto M. Droplet digital PCR for minimal residual disease detection in mature lymphoproliferative disorders. Methods Mol Biol. 2018;1768: 229-256.

31. Scherer F, Kurtz DM, Newman AM, et al Distinct biological subtypes and patterns of genome evolution in lymphoma revealed by circulating tumor DNA. Sci Transl Med. 2016;8(364):364ra155.

32. Scherer F, Kurtz DM, Diehn M, Alizadeh AA. High-throughput sequencing for noninvasive disease detection in hematologic malignancies. Blood. 2017;130(4):440-452.

33. Camus V, Jardin F, Tilly H. The value of liquid biopsy in diagnosis and monitoring of diffuse large B-cell lymphoma: recent developments and future potential. Expert Rev Mol Diagn. 2017;17(6):557-566.

34. Delfau-Larue $\mathrm{MH}$, van der Gucht A, Dupuis J, et al. Total metabolic tumor volume, circulating tumor cells, cell-free DNA distinct prognostic value in follicular lymphoma. Blood Adv. 2018;2(7):807-816.

35. Luskin MR, Murakami MA, Manalis SR Weinstock DM. Targeting minimal residual disease: a path to cure? Nat Rev Cancer 2018;18(4):255-263

36. Kolstad A, Pedersen LB, Eskelund CW, et al. Molecular monitoring after autologous stem cell transplantation and preemptive rituximab treatment of molecular relapse; results from the mordic mantle cell lymphoma studies (MCL2 and MCL3) with median follow-up of 8.5 years. Biol Blood Marrow Transplant. 2017;23(3):428-435.

37. Andersen NS, Pedersen LB, Laurell A, et al Pre-emptive treatment with rituximab of molecular relapse after autologous stem cell transplantation in mantle cell lymphoma. Clin Oncol. 2009;27(26):4365-4370.

38. Rummel MJ, Knauf W, Goener M, et al Two years rituximab maintenance vs. observation after first line treatment with bendamustine plus rituximab (B-R) in patients with mantle cell lymphoma: first results of a prospective, randomized, multicenter phase II study (a subgroup study of the StiL NHL7-2008 MAINTAIN trial). J Clin Oncol. 2016;34(15_suppl):7503. 